\title{
DENSITY ESTIMATE IN SMALL TIME FOR JUMP PROCESSES WITH SINGULAR LÉVY MEASURES
}

\author{
Dedicated to Professor Mamoru Kanda on his sixtieth birthday
}

\author{
YASUSHI ISHIKAWA
}

(Received July 12, 1999, revised July 31, 2000)

\begin{abstract}
We consider the asymptotic behaviour of the transition density for processes of jump type as the time parameter $t$ tends to 0 . We use Picard's duality method, which allows us to obtain the lower and upper bounds of the density even for the case where the support of Lévy measure is singular. The main result is that, under certain restrictions, the density behaves in polynomial order or may decrease in exponential order as $t \rightarrow 0$ according to geometrical conditions of the objective points.
\end{abstract}

Introduction. Let $x_{t}(x)$ be a $d$-dimensional jump process given by the stochastic differential equation

$$
x_{t}(x)=x+\int_{0}^{t} b\left(x_{s}(x)\right) d s+\sum_{s \leq t}^{c} \gamma\left(x_{s-}(x), \Delta z(s)\right),
$$

where $z(s)$ denotes an $\boldsymbol{R}^{d}$-valued Lévy process of jump type, $\gamma(x, \zeta)$ is a function from $\boldsymbol{R}^{d} \times \boldsymbol{R}^{d}$ to $\boldsymbol{R}^{d}$ and $\sum_{s \leq t}^{c}$ denotes the compensated sum (cf. Section 1).

Picard [18] has developed a calculus for showing the existence and the regularity of the transition density $p_{t}(x, y)$ for $x_{t}(x)$ (as the density of the law of the process $x_{t}(x)$ ), which is applicable to the case where the Lévy measure of the driving process $z(s)$ is not necessarily smooth. He uses a method of adding and subtracting Dirac masses on the Poisson time-space state space (called duality method), instead of previous methods using perturbations in space or time directions developped by [1], [2], [3] and [21] for instance. This method has, in particular, an advantage that it can be applied to the case of singular Lévy measures (e.g., it is countably supported).

In this note, we will study the asymptotic behavior of $p_{t}(x, y)$, for given $x$ and $y$, as $t \rightarrow 0$ under the condition that $z(s)$ has a singular Lévy measure. In particular, its support may be discrete ( $\mu$ is the sum of point masses.) We shall extend the type of processes treated in [19], [20], and apply the method of Picard. The present study may also be viewed as a continuation and an improvement of the same subject in [7], [8], [9] and [10]. Indeed it will be seen, as in those previous results, that the geometrical quantity $\alpha(x, y)$ attached to the support of the Lévy measure plays an important role in estimating $p_{t}(x, y)$ for accessible 
points $y$ (see Section 1 for the definition). Namely, if $y$ is accessible, then we have

$$
p_{t}(x, y) \asymp t^{\alpha(x, y)-d / \beta} \quad \text { as } t \rightarrow 0 .
$$

If $y$ is not accessible, then the density does not decay in polynomial order of $t$ as $t \rightarrow 0$ and may decrease in exponential order (see Theorem 2(b)). The resulting formula in this exponential case is very close to the one that Picard obtained in the case $d=1$, and $x_{t}(x)$ is a Lévy process.

We organize this note as follows. In Section 1 we state our results under necessary assumptions. In Section 2, we recall fundamental lemmas (Lemmas 2.1, 2.2) due to Picard, and provide several examples using these lemmas.

In Section 3 we prove Theorem 2 by using previous lemmas. More precisely, for the lower bound (Lemma 2.1), we use a point of view similar to that used in [20], [8] and [10] (it is called as a method of skelton trajectories in [10]). That is, we approximate trajectories which may have positive law by some principal trajectories given by a certain Markov chain. To obtain the upper bound (Lemma 2.2), however, this strategy is not sufficient to treat all the points of positive density. We introduce to this end, according to [20], perturbations $\left(\varphi_{n}\right)$ of the Markov chain above. This enables the estimations from above at all points which may have positive densities, and gives a strong result in the scheme of approximation of jump-type processes by Markov chains compared with [15]. The lower bound for the case of Theorem 2(b) (asymptotically accessible case) is not obtained yet. This will be studied in a subsequent paper.

A part of this work was done at Université Blaise Pascal (Clermont-Ferrand, France) in the summer of 1997 , on leave from University of Tsukuba. The author would like to thank Professor J. Picard for his hospitality and stimulative discussions during the stay. $\mathrm{He}$ is grateful also to the anonymous referee for the careful reading of the first version of this article, and for giving useful comments and advices.

1. Statement of Results. Let $z(s)$ be an $\boldsymbol{R}^{d}$-valued Lévy process with Lévy measure $\mu(d \zeta): \int_{\boldsymbol{R}^{d}}\left(|\zeta|^{2} \wedge 1\right) \mu(d \zeta)<+\infty$. That is, the characteristic function $\psi_{t}$ is given by

$$
\psi_{t}(\xi)=E\left[e^{i \xi \cdot z(t)}\right]=\exp \left(i t \xi \cdot c+t \int\left(e^{i \xi \cdot \zeta}-1-i \xi \cdot \zeta 1_{\{|\zeta| \leq 1\}}\right) \mu(d \zeta)\right) .
$$

We may write

$$
z(s)=c s+\int_{0}^{s} \int_{\boldsymbol{R}^{d} \backslash\{0\}} \zeta\left(N(d u d \zeta)-d u 1_{\{|\zeta| \leq 1\}} \cdot \mu(d \zeta)\right),
$$

where $N$ is a Poisson random measure with mean $d u \times \mu(d \zeta)$. Let $\gamma(x, \zeta): \boldsymbol{R}^{d} \times \boldsymbol{R}^{d} \rightarrow \boldsymbol{R}^{d}$ and $b(x): \boldsymbol{R}^{d} \rightarrow \boldsymbol{R}^{d}$ be $C^{\infty}$-functions, whose derivatives of all orders are bounded, satisfying $\gamma(x, 0)=0$.

We carry out our study in the probability space $\left(\Omega,\left(\mathcal{F}_{t}\right)_{t \geq 0}, P\right)$, where $\Omega=D\left[\boldsymbol{R}^{+}, \boldsymbol{R}^{d}\right]$ (Skorohood space), $\left(\mathcal{F}_{t}\right)_{t \geq 0}=$ filtration generated by $z(s)$, and $P=$ probability measure on $\omega$ of $z(s)$. That is, $\mu(d \zeta)=P(z(s+d s) \in d \zeta \mid z(s)) / d s$. 
Consider the following SDE

$$
x_{t}(x)=x+\int_{0}^{t} b\left(x_{s}(x)\right) d s+\sum_{s \leq t}^{c} \gamma\left(x_{s-}(x), \Delta z(s)\right),
$$

where $\sum^{c}$ denotes the compensated sum. That is,

$$
\sum_{s \leq t}^{c} \gamma(x, \Delta z(s))=\lim _{\varepsilon \rightarrow 0}\left\{\sum_{s \leq t,|\Delta z(s)| \geq \varepsilon} \gamma(x, \Delta z(s))-\int_{0}^{t} d s \int_{|\zeta| \geq \varepsilon} \gamma(x, \zeta) \mu(d \zeta)\right\} .
$$

Equivalently, we may write $x_{t}(x)$ as

$$
\begin{aligned}
x_{t}(x)= & x+\int_{0}^{t} b^{\prime}\left(x_{s}(x)\right) d s+\int_{0}^{t} \int_{|\zeta| \leq 1} \gamma\left(x_{s-}(x), \zeta\right) \tilde{N}(d s d \zeta) \\
& +\int_{0}^{t} \int_{|\zeta|>1} \gamma\left(x_{s-}(x), \zeta\right) N(d s d \zeta),
\end{aligned}
$$

where $\tilde{N}$ denotes the compensated Poisson random measure: $\tilde{N}(d s d \zeta)=N(d s d \zeta)-$ $d s \mu(d \zeta), b^{\prime}(x)=b(x)-\int_{|\zeta| \geq 1} \gamma(x, \zeta) \mu(d \zeta)$, where the integrability of $\gamma(x, \zeta)$ with respect to $1_{\{|\zeta| \geq 1\}} \cdot d \mu(\zeta)$ is assumed. We remark that

$$
\gamma(x, \zeta)=\frac{\partial \gamma}{\partial \zeta}(x, 0) \zeta+\tilde{\gamma}(x, \zeta)
$$

for some $\tilde{\gamma}(x, \zeta)=o(|\zeta|)$ as $\zeta \rightarrow 0$.

Throughout this paper we assume the following four assumptions (A.1)-(A.4):

(A.1) The Lévy measure satisfies that there exist some $0<\beta<2$ and positive $C_{1}, C_{2}$ such that as $\rho \rightarrow 0$,

$$
C_{1} \rho^{2-\beta} I \leq \int_{|\zeta| \leq \rho} \zeta \zeta^{*} \mu(d \zeta) \leq C_{2} \rho^{2-\beta} I
$$

where $\zeta=\left(\zeta_{1}, \ldots, \zeta_{d}\right)$ is considered as a column vector and hence inequalities are in the sense of matricies. Or equivalently, for all $u \in S^{d-1}$

$$
\int_{|\zeta| \leq \rho}\langle\zeta, u\rangle^{2} \mu(d \zeta) \asymp \rho^{2-\beta}
$$

as $\rho \rightarrow 0$. (Here $\asymp$ means the quotient of the two sides is bounded away from zero and above as $\rho \rightarrow 0$.)

(A.2) We further make the following assumptions in case $0<\beta \leq 1$ in the above:

(2-a) If $0<\beta<1$, we assume that $c=\int_{|\zeta| \leq 1} \zeta \mu(d \zeta), b=0$ and for all $u \in S^{d-1}$

$$
\int_{\{|\zeta| \leq \rho\}}\langle\zeta, u\rangle^{2} 1_{\{\langle\zeta, u\rangle>0\}}(\zeta) \mu(d \zeta) \asymp \rho^{2-\beta}
$$

as $\rho \rightarrow 0$.

(2-b) If $\beta=1$, then

$$
\limsup _{\varepsilon \rightarrow 0}\left|\int_{\{\varepsilon<|\zeta| \leq 1\}} \zeta \mu(d \zeta)\right|<+\infty
$$


(A.3) (3-a) For any $p \geq 2$ and any $k \in N^{d} \backslash\{0\}$,

$$
\int|\gamma(x, \zeta)|^{p} d \mu(\zeta) \leq C(1+|x|)^{p}, \quad \sup _{x} \int\left|\frac{\partial^{k} \gamma}{\partial x^{k}}(x, \zeta)\right|^{p} d \mu(\zeta)<+\infty .
$$

(3-b) There exists $\delta>0$ such that

$$
\inf \left\{y^{*}\left(\frac{\partial \gamma}{\partial \zeta}(x, 0)\right)\left(\frac{\partial \gamma}{\partial \zeta}(x, 0)\right)^{*} y ; x \in \boldsymbol{R}^{d}\right\} \geq \delta|y|^{2}
$$

on $\boldsymbol{R}^{d}$.

(A.4) We assume that for some $C>0$,

$$
\inf _{x \in \boldsymbol{R}^{d}, \zeta \in \operatorname{supp} \mu}\left|\operatorname{det}\left(I+\frac{\partial \gamma}{\partial x}(x, \zeta)\right)\right|>C .
$$

The condition (1.7) guarantees the existence of the flow $\phi_{s t}(x)(\omega): \boldsymbol{R}^{d} \rightarrow \boldsymbol{R}^{d}, x_{s}(x) \mapsto$ $x_{t}(x)$ of diffeomorphisms for all $0<s \leq t$ (cf. [13]).

Equation (1.1) (resp. (1.2)) has a unique solution. This follows from the fact that (1.1) can be written in the canonical form (in Ito integral)

$$
d x_{t}(x)=d \Theta_{t}\left(x_{t-}(x)\right), \quad x_{0}(x)=0,
$$

where

$$
\Theta_{t}(y)=b^{\prime}(y) t+\int_{0}^{t}\left\{\int_{|\zeta| \leq 1} \gamma(y, \zeta) \tilde{N}(d s d \zeta)+\int_{|\zeta|>1} \gamma(y, \zeta) N(d s d \zeta)\right\},
$$

and that the equation (1.8) has a unique solution due to [4, Theorem 2.1] (cf. [12, Theorem 3.1]). Furthermore, due to the inversibility assumption (A.4), there exists a stochastic flow of diffeomorphisms, denoted by $\phi_{s, t}(s<t): \boldsymbol{R}^{d} \rightarrow \boldsymbol{R}^{d}$, such that $x_{t}(x)=\phi_{s, t}\left(x_{s}(x)\right)$, which is inversible ([13, Section 1], [1, Lemma 7-27]; see also [22, Theorem V65] for the simple case $\gamma(x, \zeta)=X(x) \zeta)$.

We cite the following fundamental result due to Picard [18].

Proposition 1.1 (cf. [18, Theorem 4.1]). Under the conditions (A.1) through (A.4), $x_{t}(x)$ has a $C_{b}^{\infty}$-density for each $t>0$, which we denote by $y \mapsto p_{t}(x, y)$.

We remark the crucial condition: for each $t>0, p \geq 1$,

$$
\begin{gathered}
\left\|\left(\operatorname{det}\left(\int_{0}^{t} \psi_{s} \psi_{s}^{*} d s\right)\right)^{-1}\right\|_{L^{p}}<+\infty \\
\text { with } \psi_{s}=\left(\frac{\partial}{\partial x} \phi_{s, t}\left(x_{s}(x)\right)\right)\left(\frac{\partial \gamma}{\partial \zeta}\left(x_{s}(x), 0\right)\right)
\end{gathered}
$$

for the existence of the density in [18] being derived from (1.6) and (1.7) by an argument similar to that in the proof of Corollary 4.4 of [18].

Let $v(d \zeta)$ be the probability measure on $\boldsymbol{R}^{d}$ given by

$$
v(d \zeta)=\frac{\left(|\zeta|^{2} \wedge 1\right) \mu(d \zeta)}{\left.\int\left(|\zeta|^{2} \wedge 1\right) \mu(d \zeta)\right)}
$$


Then $d v \sim d \mu$ and $d v / d \mu$ is globally bounded from above.

The following result is an extension of Theorem 1 in [20] to the above mentioned type of processes.

THEOREM 1 (general upper bound: cf. Picard [20]). The density $p_{t}(x, y)$ satisfies the following estimate:

$$
\text { (a) } \sup _{x, y} p_{t}(x, y) \leq C_{0} t^{-d / \beta} \quad \text { as } t \rightarrow 0
$$

$$
p_{t}(x, x) \asymp t^{-d / \beta} \quad \text { as } t \rightarrow 0 \text { uniformly in } x .
$$

(b) For all $k \in N^{d}$ there exists $C_{k}>0$ such that

$$
\sup _{x, y}\left|p_{t}^{(k)}(x, y)\right| \leq C_{k} t^{-(|k|+d) / \beta} \quad \text { as } t \rightarrow 0,
$$

where $p^{(k)}$ denotes the $k$-th derivative with respect to $y$.

We can give examples that the supremum in (a) is in fact attained on the diagonal $\{x=y\}$ (diagonal estimate):

$$
p_{t}(x, x) \asymp t^{-d / \beta} \quad \text { as } t \rightarrow 0
$$

uniformly in $x$ (see [7, Section 2-4]). The estimate above is more exact than the one by Hoh-Jacob [5] using functional analytic methods: there exist $C>0, v \in(1, \infty)$ such that

$$
\sup _{x, y} p_{t}(x, y) \leq C t^{-v} \quad \text { as } t \rightarrow 0
$$

Consider a series of functions $\left(A_{n}\right)_{n=0}^{\infty}, A_{n}: \boldsymbol{R}^{d \times(n+1)} \rightarrow \boldsymbol{R}^{d}$, defined by $A_{0}(x)=x$ and $A_{n+1}\left(x, x_{1}, \ldots, x_{n+1}\right)=A_{n}\left(x, x_{1}, \ldots, x_{n}\right)+\gamma\left(A_{n}\left(x, x_{1}, \ldots, x_{n}\right), x_{n+1}\right)$. Fix $x \in \boldsymbol{R}^{d}$. We put $\mathcal{S}_{n}$ to be the support of the image measure of $\mu^{\otimes n}$ by the mapping $\left(x_{1}, \ldots, x_{n}\right) \mapsto$ $A_{n}\left(x, x_{1}, \ldots, x_{n}\right)$, and $\mathcal{S} \equiv \bigcup_{n} \mathcal{S}_{n}$.

DEFINITION 1.2 (accessible points). Points in $\mathcal{S}$, regarded as points in $\boldsymbol{R}^{d}$, are called accessible points. Points in $\overline{\mathcal{S}} \backslash \mathcal{S}$ are called asymptotically accessible points.

Intuitively accessible points are those points which can be reached by $x_{t}(x)$ using only by a finite number of jumps of $z(s)$. We remark that $\mathcal{S}$ is not necessarily closed, although each $\mathcal{S}_{n}$ is so.

We define for each $x$ the mapping $H_{x}: \operatorname{supp} v \rightarrow P_{x} \equiv x+\{\gamma(x, \zeta) ; \zeta \in \operatorname{supp} v\}$ by $\zeta \mapsto x+\gamma(x, \zeta)$. Let $P_{x}^{(n)}=\left\{y \in P_{z_{n-1}} ; z_{1} \in P_{x}, z_{i} \in P_{z_{i-1}}, i=2, \ldots, n-1\right\}$, $n=1,2, \ldots\left(z_{0}=x\right)$. Then $P_{x}^{(1)}=P_{x}$, and $P_{x}^{(n)}$ can be interpreted as points which can be reached from $x$ by $n$ jumps along the trajectory $x_{t}(x)$. Given $x, y \in \boldsymbol{R}^{d}(y \neq x)$, let $\alpha(x, y)$ be the minimum number $l$ such that $y \in P_{x}^{(l)}$ if such $l$ exists, and put $\alpha(x, y)=+\infty$ if not. Or equivalently, $\alpha(x, y)=\inf \left\{n ; y \in \bigcup_{k \leq n} \mathcal{S}_{k}\right\}$.

Now we introduce a concrete "singular" Lévy measure of $z(s)$, which has already described in [23, Example 3.7], and in [2, Section 2]. Let $\mu(d \zeta)=\sum_{n=0}^{\infty} k_{n} \delta_{a_{n}}(d \zeta)$ be the $d$-dimensional Lévy measure such that $\left(a_{n} ; n \in N\right)$ and $\left(k_{n} ; n \in N\right)$ are sequences of points in $\boldsymbol{R}^{d}$ and real numbers, respectively, satisfying 
(i) $\left|a_{n}\right|$ decreases to 0 as $n \rightarrow \infty$,

(ii) $k_{n}>0$,

(iii) $\sum_{n=0}^{\infty} k_{n}\left|a_{n}\right|^{2}<+\infty$.

For this Lévy measure, we can show the unique existence of the solution $x_{t}(x)$ of (1.2) ([23, Theorems 1.1, 2.1]), and the existence of the density under the assumptions (A.1)-(A.4). We further assume that

$$
N=N(t) \equiv \max \left\{n ;\left|a_{n}\right|>t^{1 / \beta}\right\} \asymp \log \left(\frac{1}{t}\right) .
$$

The next theorem (b) can be viewed as an extension of Proposition 5.2 in [19].

THEOREM 2. Let $y \neq x$.

(a) Assume $y \in \mathcal{S}$, that is, $\alpha(x, y)<+\infty$. Then we have

$$
p_{t}(x, y) \asymp t^{\alpha(x, y)-d / \beta} \quad \text { as } t \rightarrow 0 .
$$

(b) Assume $y \in \overline{\mathcal{S}} \backslash \mathcal{S}(\alpha(x, y)=+\infty)$. Suppose $b(x) \equiv 0$ and let $\beta^{\prime}>\beta$. Then $\log p_{t}(x, y)$ is bounded from above by the expression of type $\Gamma=\Gamma(t)$ :

$$
\Gamma \equiv-\min \sum_{n=0}^{N}\left(w_{n} \log \left(1 /\left(k_{n}\right)\right)+\log \left(w_{n} !\right)\right)+O(\log (1 / t) \log \log (1 / t))
$$

as $t \rightarrow 0$. Here the minimum is taken with respect to all choice of $a_{0}, \ldots, a_{N}$ by $\xi_{n}$ for $n=1,2, \ldots, n_{1}$ and $n_{1} \in N$ such that

$$
\left|y-A_{n_{1}}\left(x, \xi_{1}, \ldots, \xi_{n_{1}}\right)\right| \leq t^{1 / \beta^{\prime}},
$$

where $w_{n}=\#$ of $a_{n}$ in the choice and $n_{1}=\sum_{n=0}^{N} w_{n}$.

We remark that in finding the above minimum the conditions (1.14) and (1.17) work complementarily. That is, as $t>0$ gets smaller, (1.17) becomes apparently more strict, whereas we may use more combinations of $a_{i}$ 's to approximate $y$ due to (1.14). Since $\beta^{\prime}>\beta$, the condition (1.17) does not prevent $\dot{A}_{n_{1}}\left(x, \xi_{1}, \ldots, \xi_{n_{1}}\right)$ from attaining to $y$ as $t \rightarrow 0$, using $a_{0}, \ldots, a_{N}$ under (1.14). We also remark that if $x$ and $a_{n}$ are rational points (e.g., $d=1$, $\left.x=0, a_{n}=2^{-n}\right)$, then the result (b) holds for almost all $y \in \overline{\mathcal{S}}(=[0,1])$ relative to the Lebesgue measure, whereas for $t>0, y \mapsto p_{t}(x, y)$ is smooth on $\overline{\mathcal{S}}$ due to Proposition 1.1.

The proof of these theorems will be given in Section 3 .

Notation. Here we sum up notation we will use throughout this note.

$z(s) \quad$ Lévy process with Lévy measure $d \mu$

$x_{s}(x)$ the jump type process driven by $z(s)$

$\tilde{z}^{r}(s) \quad$ Lévy process defined by $\tilde{z}^{r}(s)=\sum_{u \leq s} \Delta z(u) 1_{\{|\Delta z(u)|>r\}}$

$z^{r}(s) \quad$ Lévy process defined by $z^{r}(s)=z(s)-\tilde{z}^{r}(s)$

2. Preliminaries. In this section, we prove two lemmas which are essential for our estimation. These lemmas are inspired by Picard [20]. Proofs of these lemmas can be derived 
easily in view of Picard [20], using several corresponding lemmas. Let $\left(\xi_{n}\right)_{n \in N}$ be an $\boldsymbol{R}^{d}$ valued series of random variables (i.i.d.) obeying the probability law $v(d \zeta)$, independent of $z(s)$.

LEMMA 2.1 (lower bound for accessible points). Let $\left(\xi_{n}\right)_{n \in N}$ be an $\boldsymbol{R}^{d}$-valued random variables (i.i.d.) obeying the probability law $v(d \zeta)$, independent of $z(s)$. We define a Markov chain $\left(U_{n}\right)_{n \in N}$ by $U_{0}=x$ and $U_{n+1}=U_{n}+\gamma\left(U_{n}, \xi_{n+1}\right), n \in N$. Assume that for $y \in \boldsymbol{R}^{d}$, there exist some $n \geq 1, \gamma=\gamma_{n} \geq 0$ and $c>0$ such that for all $\varepsilon \in(0,1], P\left(\left|U_{n}-y\right| \leq\right.$ $\varepsilon) \geq c \varepsilon^{\gamma}$. Then we have

$$
p_{t}(x, y) \geq C t^{n+(\gamma-d) / \beta} \quad \text { as } t \rightarrow 0 .
$$

We notice that the lower bound in R.H.S. depends on $\left(n, \gamma_{n}\right)$. Put $g(x, d z)=d\left(H_{x}^{*} v\right)(z)$, $z \in P_{x} \backslash\{x\}$, where $H_{x}^{*} v=v \circ H_{x}^{-1}$ (cf. Section 1). Then we have an expression of the probability above:

$$
P\left(\left|U_{n}-y\right| \leq \varepsilon\right)=\int_{P_{x}} \cdots \int_{P_{z_{n-1}}} 1_{\left\{z_{n} ;\left|z_{n}-y\right| \leq \varepsilon\right\}}\left(z_{n}\right) g\left(x, d z_{1}\right) \cdots g\left(z_{n-1}, d z_{n}\right)
$$

(cf. [8]). Hence the condition $P\left(\left|U_{n}-y\right| \leq \varepsilon\right) \geq c \varepsilon^{\gamma}$ implies:

$y$ can be attained with the singular Lévy measure (dim supp $v=0)$ if $\gamma=0$.

Let $\left(\varphi_{n}\right)_{n \in \boldsymbol{N}}$ be a series of smooth functions: $\boldsymbol{R}^{d} \rightarrow \boldsymbol{R}^{d}$. We define another Markov chain $\left(V_{n}\right)_{n \in N}$ by $V_{0}=\varphi_{0}(x)$ and $V_{n+1}=V_{n}+\left(\varphi_{n+1} \circ \gamma\right)\left(V_{n}, \xi_{n+1}\right)$. Furthermore we define the series of real numbers $\left(\Phi_{n}\right)_{n \in N}$ by

$$
\Phi_{n} \equiv \sup _{k \leq n, y \in \boldsymbol{R}^{d}}\left(\left|\varphi_{k}(y)-y\right|+\left|\varphi_{k}^{\prime}(y)-I\right|\right) .
$$

Under these preparations we have

LEMMA 2.2 (upper bound). Choose $y \neq x$. Assume there exist a sequence $\left(\gamma_{n}\right)_{n \in N}$, $\gamma_{n} \in[0, \infty]$, and a non-decreasing sequence $\left(K_{n}\right)_{n \in N}, K_{n}>0$, satisfying the following condition: for each $n$ and for any $\left(\varphi_{k}\right)_{k=0}^{n}$ satisfying $\Phi_{n} \leq K_{n}, V_{n}$ defined as above satisfies that with some $C_{n}>0$

$$
\text { if } \gamma_{n}<+\infty \text {, then } P\left(\left|V_{n}-y\right| \leq \varepsilon\right) \leq C_{n} \varepsilon^{\gamma_{n}} \text { for all } \varepsilon>0 \text {, }
$$

and

$$
\text { if } \gamma_{n}=+\infty \text {, then } P\left(\left|V_{n}-y\right| \leq \varepsilon\right)=0 \text { for } \varepsilon>0 \text { small. }
$$

Furthermore, if we put $\Gamma \equiv \min _{n}\left(n+\left(\gamma_{n}-d\right) / \beta\right)$, then we have:

(i) If $\Gamma<+\infty$, then $p_{t}(x, y)=O\left(t^{\Gamma}\right)$ as $t \rightarrow 0$.

(ii) If $\Gamma=+\infty$, then for any $n \in N p_{t}(x, y)=o\left(t^{n}\right)$ as $t \rightarrow 0$.

Note that $\Gamma$ depends implicitly on the choice of $\left(K_{n}\right)$. However, for each $n$, bigger $\gamma_{n}$ gives a better upper bound.

Given perturbations $\left(\varphi_{j}\right)$, we define $Q_{x}^{(0)}=\left\{\varphi_{0}(x)\right\}$ and the sequence $\left(Q_{x}^{(n)}\right)$ successively by

$$
Q_{x}^{(n)} \equiv\left\{z_{n-1}+\left(\varphi_{n} \circ \gamma\right)\left(z_{n-1}, \zeta\right) ; \zeta \in \operatorname{supp} \nu, z_{n-1} \in Q_{x}^{(n-1)}\right\}
$$


for $n=1,2, \ldots$. Hence the set $Q_{x}^{(n)}$ can be interpreted as the points which can be reached from $x$ by $V_{n}$, and we have

$$
\begin{aligned}
& P\left(\left|V_{n}-y\right| \leq \varepsilon\right) \\
& \quad=\int_{P_{\varphi_{0}(x)}} \cdots \int_{P_{\varphi_{n-1}\left(z_{n-1}\right)}} 1_{\left\{z_{n} ;\left|\varphi_{n}\left(z_{n}\right)-y\right| \leq \varepsilon\right\}}\left(z_{n}\right) g\left(\varphi_{0}(x), d z_{1}\right) \cdots g\left(\varphi_{n-1}\left(z_{n-1}\right), d z_{n}\right)
\end{aligned}
$$

for $n=0,1,2, \ldots$. We remark that if $y \notin P_{x}^{(n)}(n \geq 1)$, then by choosing $\varphi_{n}$ such that the size $\Phi_{n}$ of perturbations is small enough, we can let $y \notin Q_{x}^{(n)}$; so that by choosing $K_{n}>0$ small and $\varepsilon>0$ small, $\gamma_{n}$ may be $+\infty$ in the above.

EXAMPLES. We give here some results which are derived from above lemmas but are not in the scope of our main result. In the following examples, we assume that the drift coefficient $b$ for $x_{t}(x)$ is identically zero, so that $x_{t}(x)$ is a martingale.

(1) First we give an example where the Lévy measure has an absolutely continuous density with respect to $d$-dimensional Lebesgue measure, treated in [8].

Consider the case $\mu(d \zeta)=h(\zeta) d \zeta$, where $h(\zeta) \in C^{\infty}\left(\boldsymbol{R}^{d} \backslash\{0\}\right)$ is symmetric and satisfies

(i) $\operatorname{supp} h(\cdot) \subset\left\{\zeta \in \boldsymbol{R}^{d} ;|\zeta| \leq c\right\}$ where $0<c \leq+\infty$,

(ii) $\int_{\boldsymbol{R}^{d} \backslash\{0\}} \min (|\zeta|, 1) h(\zeta) d \zeta<+\infty$,

(iii) $h(\zeta)=a(\zeta /|\zeta|)|\zeta|^{-d-\alpha}, \alpha \in(0,1)$, in a neighbourhood of the origin with $a(\cdot) \in$ $C^{\infty}\left(S^{d-1}\right)$.

From these assumptions, we can choose $v$ to have a bounded density. As in [8, Section 2], we can show that $U_{1}$, and hence $U_{n}$, have bounded densities, so that the assumption holds with the index $\gamma=d$ in Lemma 2.1 with $n \geq \alpha(x, y)$. Here $\alpha(x, y)$ is the integer introduced in Section 1. Furthermore, the assumption in Lemma 2.2 is satisfied with $\gamma_{n}=d$ and with $K_{n}$ small if $\alpha(x, y) \leq n$, and $\gamma_{n}=+\infty$ if $\alpha(x, y)>n$ (choose $\varphi_{n}=i d$ ). Hence we have $\Gamma=\alpha(x, y)$, and $p_{t}(x, y) \asymp t^{\alpha(x, y)}$ as $t \rightarrow 0$.

(2) Our next example is the one treated in [7] and [10]. Let $\mu$ be the $d$-dimensional Lévy measure

$$
\mu(d \zeta)=h\left(\zeta_{1}\right) \otimes \cdots \otimes h\left(\zeta_{d}\right) d \zeta_{1} \cdots d \zeta_{d}
$$

where each $h \in C^{\infty}(\boldsymbol{R} \backslash\{0\})$ satisfies

(i) $\operatorname{supp} h(\cdot) \subset[-c, c] \backslash\{0\}$ where $0<c \leq+\infty$,

(ii) $h>0$ on $(-c, c) \backslash\{0\}$, and

$$
h(\zeta)=\left(a^{-} 1_{(-\infty, 0)}(\zeta)+a^{+} 1_{(0,+\infty)}(\zeta)\right)|\zeta|^{-1-\beta}
$$

in some neighborhood of the origin for some $\beta \in(0,2)$ and $a^{+} \geq 0, a^{-} \geq 0, a^{+}+a^{-}=1$. We choose $\gamma(x, \zeta)$ to be $\gamma(x, \zeta)=\left(X_{1}(x), \ldots, X_{d}(x)\right)^{t} \zeta$, where $X_{j}$ are $C^{\infty} \boldsymbol{R}^{d}$-valued functions, whose derivatives of all orders are bounded, such that $X(x) \equiv\left(X_{1}(x), \ldots, X_{d}(x)\right)$ forms a basis of $T_{x}\left(\boldsymbol{R}^{d}\right), x \in \boldsymbol{R}^{d}$. In this case we can show the existence of a smooth density 
(cf. [7]). The measure $g(x, d \zeta)$ is given by

$$
\int f(z) g(x, d \zeta)=C \int f\left(x+\boldsymbol{X}(x)^{t} \zeta\right) h\left(\zeta_{1}\right) \cdots h\left(\zeta_{d}\right) d \zeta_{1} \cdots d \zeta_{d}
$$

For simplicity, we assume that $\operatorname{supp} h=[-1,1](c=1)$ and $X_{i}={ }^{t}(0, \ldots, 0,1,0, \ldots$, $0)$, where the component 1 is at $i$-th coordinate, so that $X=I_{d}$. Let $x=(0, \ldots, 0)$. We first choose $y \neq x$ be on $(\boldsymbol{R} \backslash\{0\}) \times\{0\}^{d-1}$; so that $\alpha(x, y)<+\infty$. By the expression (1.12) we have

$$
\begin{gathered}
P\left(\left|U_{n}-y\right| \leq \varepsilon\right) \asymp \varepsilon^{1} \quad \text { as } \varepsilon \rightarrow 0 \text { if } n \geq \alpha(x, y), \\
P\left(\left|U_{n}-y\right| \leq \varepsilon\right)=0 \text { if } n<\alpha(x, y) .
\end{gathered}
$$

Hence we can choose $\gamma=1$ for the lower bound (cf. [7, Section 2-4]):

$$
p_{t}(x, y) \geq C t^{\alpha(x, y)-(d-1) / \beta} \quad \text { as } t \rightarrow 0 .
$$

For the upper bound, we may have for some $\left(\varphi_{n}\right)$ for which $\Phi_{n}$ is small, that $\gamma_{n}=+\infty$ for $n<\alpha(x, y)$ by the reason given after (2.6). For $n \geq \alpha(x, y)$, we have from (2.9) that $\gamma_{n}=1$ for some $K_{n}$ small. Hence $\Gamma=\alpha(x, y)-(d-1) / \beta$. Therefore

$$
p_{t}(x, y) \asymp t^{\alpha(x, y)-(d-1) / \beta} \quad \text { as } t \rightarrow 0 .
$$

In [10] we gave an upper bound $C t^{\alpha(x, y)(1-(d-1) / \beta)}$ for this case.

Next we choose $y$ in $(\boldsymbol{R} \backslash\{0\})^{d}$. Then, similarly to the above, $\alpha(x, y)<+\infty$, and we have:

$$
\begin{gathered}
P\left(\left|U_{n}-y\right| \leq \varepsilon\right) \asymp \varepsilon^{d} \quad \text { as } \varepsilon \rightarrow 0 \text { if } n \geq \alpha(x, y), \\
P\left(\left|U_{n}-y\right| \leq \varepsilon\right)=0 \quad \text { if } n<\alpha(x, y), \\
p_{t}(x, y) \asymp t^{\alpha(x, y)} \quad \text { as } t \rightarrow 0 .
\end{gathered}
$$

3. Proof of Theorem 1. First we prove two lemmas.

LEMMA 3.1. Let $\phi_{s, t}$ be the stochastic flow of diffeomorphisms generated by $x_{t}(x)$. Then for any $q \geq 1, k \in N^{d} \backslash\{0\}$

$$
\sup _{(t, y) \in[0,1] \times \boldsymbol{R}^{d}} E\left[\sup _{s \leq t}\left|\left(\phi_{s, t}^{-1}\right)^{(k)}(y)\right|^{q}\right]<+\infty .
$$
SDE

PROOF. Consider the reversed process $Z_{s}=\phi_{(t-s)-, t}^{-1}$. Then $Z_{s}$ satisfies the backward

$$
\boldsymbol{Z}_{s}=y-\int_{0}^{s} b\left(\boldsymbol{Z}_{u}\right) d u+\sum_{u \leq s}^{c} \gamma_{0}\left(\boldsymbol{Z}_{u-}, \Delta V_{u}\right),
$$

where $V_{u}=z(t)-z((t-u)-), \gamma_{0}(x, \zeta)$ satisfies that for $\mu$-a.e. $\zeta, x \mapsto x+\gamma_{0}(x, \zeta)$ is the inverse map of $x \mapsto x+\gamma(x, \zeta)$. Then [4, Section 3 (3.7)] implies (3.1). 
LEMMA 3.2. Let $h>0$ and $k \in N^{d}$. Denote by $p_{r, t}(x, y)$ the density of $x_{t}^{r}(x)$, where $x_{t}^{r}(x)$ is defined as (1.1) with $z(s)$ replaced by $z^{r}(s)$. Then

$$
\left|p_{r, r^{\beta} h}^{(k)}(x, y)\right| \leq C_{k h} r^{-(|k|+d)} \text { for } 0<r \leq 1 .
$$

PROOF. Let $h>0$ and $r>0$. We put $\bar{x}_{h}^{r}(x) \equiv(1 / r) x_{r^{\beta} h}^{r}(x) . \bar{x}_{h}^{r}(x)$ satisfies

$$
\bar{x}_{h}^{r}(x)=\int_{0}^{h} b_{r}\left(\bar{x}_{h^{\prime}}^{r}(x)\right) d h^{\prime}+\sum_{h^{\prime} \leq h}^{c} \gamma_{r}\left(\bar{x}_{h^{\prime}-}^{r}(x), \Delta \bar{z}^{r}\left(h^{\prime}\right)\right)+\frac{1}{r} x,
$$

where $\bar{z}^{r}(t) \equiv(1 / r) z^{r}\left(r^{\beta} t\right), b_{r}(y) \equiv r^{\beta-1} b(r y)$ and $\gamma_{r}(x, \zeta) \equiv(1 / r) \gamma(r x, r \zeta)$. The assumption (A.2) (2-b) is used here to guarantee that the scaled drift parameter $c_{r}=$ $r^{\beta-1}\left(c-\int_{\{r<|\zeta| \leq 1\}} \zeta \mu(d \zeta)\right)$ is finite. Then, by the definition of $h \mapsto \bar{x}_{h}^{r}(x)$,

$$
P\left(\bar{x}_{h}^{r}(x) \in d(y / r) \mid \bar{x}_{0}^{r}(x)=x / r\right)=P\left(x_{r^{\beta} h}^{r}(x) \in d y\right) .
$$

On the other hand, $y \mapsto b_{r}(y)$ is in $C_{b}^{\infty}$ (we use here the assumption for the case $\beta<1$ ). $\gamma_{r}(x, \zeta)$ satisfies the assumptions (A.1) through (A.4) uniformly in $r$. Hence by Proposition 1.1, $\left(\bar{x}_{h}^{r}(x) ; r>0\right)$ have $C_{b}^{\infty}$-densities $\bar{p}_{h}(x / r, y: r)$, which are uniformly bounded with respect to $r>0$. Hence, by the above relation,

$$
p_{r, r^{\beta} h}(x / r, y)=\frac{1}{r^{d}} \bar{p}_{h}(x / r, y / r: r) .
$$

This implies

$$
\left|p_{r, r^{\beta} h}(x / r, y)\right| \leq C_{h} r^{-d} .
$$

Estimates for the derivatives with respect to $y$ follows by differetiating the equality.

We are in a position to prove Theorem 1 (first part of (a) and (b)). We follow here Picard [20].

Let $I_{n}(t)=\left[t_{n}, t_{n+1}\right] \equiv\left[t\left(1-2^{-n+1}\right), t\left(1-2^{-n}\right)\right], n=1,2, \ldots$, and put the random variable $N \equiv \min \left\{n ; \sup _{u \in I_{n}(t)}|\Delta z(u)| \leq r\right\}$, where $r=r(n, t)=\gamma t^{1 / \beta} 2^{-n / \beta}$. Then we have

$$
\begin{aligned}
P(N>n) & \left.=\prod_{k=1}^{n} P\left(\sup \left\{|\Delta z(u)| ; u \in I_{k}(t)\right\}>r(k, t)\right\}\right) \\
& =\prod_{k=1}^{n}\left(1-\exp \left(-\left|I_{k}(t)\right| \mu\{|\zeta|>r(k, t)\}\right)\right) \\
& \leq \prod_{k=1}^{n}\left(\left|I_{k}(t)\right| \mu\{|\zeta|>r(k, t)\}\right)
\end{aligned}
$$

by the Poisson representation of $z(s)$. Since $\mu\{|\zeta|>\rho\} \asymp \rho^{-\beta}$ ((A.1), (A.2)), $\mu\{|\zeta|>r\} \leq$ $c \gamma^{-\beta} 2^{k} t^{-1}$, and hence

$$
P(N>n) \leq c^{n} \gamma^{-n \beta}
$$


We put the interval $I_{N}=\left[S^{\prime}, S\right]$. We denote by $\psi_{n}: \boldsymbol{R}^{d} \rightarrow \boldsymbol{R}^{d}$ the flow of $z^{r(n, t)}(s)$ from $t_{n}$ to $t_{n+1}$ (that is, $\left.x_{t_{n+1}}^{r(n, t)}(x)=\psi_{n}\left(x_{t_{n}}^{r(n, t)}\right)\right)$, so that $x_{S}(x)=\psi_{N}\left(x_{S^{\prime}}(x)\right)$. The truncated process $x_{t}^{r}(x)$ has a $C_{b}^{\infty}$ density $p_{r, t}(x, y)$ by Proposition 1.1. Hence

$$
P\left(\psi_{n}\left(x^{\prime}\right) \in d y\right)=p_{r(n, t),\left|I_{n}(t)\right|}\left(x^{\prime}, y\right) d y .
$$

Then

$$
\begin{aligned}
P\left(x_{S}(x) \in d y \mid N=n, x_{S^{\prime}}(x)=x^{\prime}\right) & =P\left(\psi_{n}\left(x^{\prime}\right) \in d y \mid N=n, x_{t_{n}}(x)=x^{\prime}\right) \\
& =P\left(\psi_{n}\left(x^{\prime}\right) \in d y\right) .
\end{aligned}
$$

On $I_{N}, z(s)$ makes no greater jumps than $\tilde{r} \equiv r(N, t)$, and hence

$$
P\left(x_{S}(x) \in d y \mid N=n, x_{S^{\prime}}(x)=x^{\prime}\right)=p_{\tilde{r},\left|I_{N}\right|}\left(x_{S^{\prime}}(x), y\right) d y .
$$

Since $\left|I_{N}\right|=t / 2^{N}=(r(N, t) / \gamma)^{\beta}$,

$$
P\left(x_{S}(x) \in d y \mid N\right) / d y=E\left[p_{\tilde{r},(\tilde{r} / \gamma)^{\beta}}\left(x_{S^{\prime}}(x), y\right) \mid N\right] .
$$

Denote by $\phi_{s, t}$ the flow of diffeomorphisms generated by $x_{t}(x)$, so that $x_{t}(x)=$ $\phi_{S, t}\left(x_{S}(x)\right)$ for $t \geq S$. Then, by changing the variable $\tilde{y}=\phi_{S, t}^{-1}(y)$ and taking the expectation with respect to $N$ in both sides of the above,

$$
p_{t}(x, y)=E\left[p_{\tilde{r},\left(\frac{\tilde{r}}{\gamma}\right)^{\beta}}\left(x_{S^{\prime}}(x), \phi_{S, t}^{-1}(y)\right) \cdot\left|\operatorname{det}\left(J\left(\phi_{S, t}^{-1}\right)(y)\right)\right|\right] .
$$

Here $J \phi^{-1}(y)=\left(\partial \phi^{-1} / \partial \zeta\right)(y)$ denotes the Jacobian matrix of $\phi^{-1}$ at $y$. We remark this change-of-variables calculation is possible, since, conditionally on $N, \phi_{S, t}$ is independent of $x_{S}(x)$ and $x_{S^{\prime}}(x)$.

Here $\sup _{(t, y) \in[0,1] \times \boldsymbol{R}^{d}} E\left[\left|\sup _{s \leq t}\right| \operatorname{det}\left(J\left(\phi_{S, t}^{-1}\right)\right)|(y)|^{q}\right]<+\infty$ for all $q \geq 1$ by Lemma 3.1 with $|k|=1$. We also have, for $h>0$,

$$
\left|p_{r, r^{\beta} h}^{(k)}(x, y)\right| \leq C_{h k} r^{-(|k|+d)}
$$

for $0<r \leq 1$ (Lemma 3.2).

Choose $h=\gamma^{-\beta}$ and $r=\tilde{r} \equiv r(N, t)$. Then we obtain by (3.3)

$$
\begin{aligned}
p_{t}(x, y) & \leq E\left[C_{\gamma} r(N, t)^{-d}\left|\operatorname{det}\left(J\left(\phi_{S, t}^{-1}\right)\right)\right|(y) \mid\right] \\
& \leq C_{\gamma} C_{J}\left\|r(N, t)^{-d}\right\|_{L^{2}(P)}=C_{\gamma}^{\prime} t^{-d / \beta}\left\|\gamma^{-d} 2^{N d / \beta}\right\|_{L^{2}(P)} .
\end{aligned}
$$

We choose $\gamma>0$ so that $\left\|\gamma^{-d} 2^{N d / \beta}\right\|_{L^{2}(P)} \leq M$, and hence $p_{t}(x, y) \leq C_{0} t^{-d / \beta}$.

The proof of the second statement of (a) is given below.

For the derivatives with respect to $y$ (case (b)), we also have from Lemmas 3.1 and 3.2 that

$$
p_{t}^{(k)} \leq C_{k, \gamma} t^{-(d+|k|) / \beta}\left\|\gamma^{-(d+|k|)} 2^{N+(d+|k|) / \beta}\right\|_{L^{2}(P)} .
$$

Again, by choosing large $\gamma$, we have the conclusion (b).

We next prove the second part of (a). We first have

LEMMA 3.3. Consider an $\boldsymbol{R}^{d}$-valued infinitely divisible random variable $\Theta_{t}$ indexed by $t>0$, and another $\boldsymbol{R}^{d}$-valued random variable $Y_{t}$. We assume that 
(i) $Y_{t}$ has a $C_{b}^{\infty}$ transition density uniformly in $t$,

(ii) $\left\|Y_{t}-\Theta_{t}\right\|_{L^{1}} \rightarrow 0$ as $t \rightarrow 0$,

(iii) for the Lévy measure $\mu_{t}$ of $\Theta_{t}$, there exist a compact $K \subset \boldsymbol{R}^{d}$, and $M>0$ such that $\operatorname{supp} \mu_{t} \subset K$, and $\int|x|^{2} \mu_{t}(d x) \leq M$ (uniformly in $t \leq 1$ ), respectively. We further assume that $\mu_{t}$ satisfies (A.1) and (A.2). Then the density of $Y_{t}$ is bounded away from zero as $t \rightarrow 0$ on any compact set.

For the proof, see [20, Lemma 6]. The conclusion essentially follows from the uniform boundedness of $\mu_{t}(d x)$.

From the assumption (A.1) we have $\mu\{|\zeta|>\rho\} \asymp \rho^{-\beta}$, and hence we have $\mu\{|\zeta|>$ $r(t)\} \leq C r^{-\beta}$. We put $A_{t} \equiv\left\{\sup _{s \leq t}|\Delta z(s)| \leq r(t)\right\}$. Since $t \cdot \mu\{|\zeta|>r(t)\} \leq C$, we have $P\left(A_{t}\right)>0$ uniformly as $t \rightarrow 0$.

On $A_{t}, z(s)=z^{r}(s), x_{t}(x)=x_{t}^{r}(x)$ on $[0, t]$. Since $x_{t}^{r}(x)$ and $A_{t}$ are independent,

$$
p_{t}(x, y) \geq P\left(A_{t}\right) \cdot P\left(x_{t}^{r}(x) \in d y\right) / d y \geq c P\left(x_{t}^{r}(x) \in d y\right) / d y
$$

with some $c>0$. Hence we have only to prove that the density of $\mathcal{X}_{t} \equiv t^{-1 / \beta}\left(x_{t}^{r}(x)-x\right)$ is bounded away from zero at 0 .

$\mathcal{X}_{t}$ has a $C_{b}^{\infty}$ density for each $t>0$ by Proposition 1.1. We shall approximate it by some infinitely divisible random variable. Put $t^{\prime} \mapsto \bar{z}^{r}\left(t^{\prime}\right)$ by $\bar{z}^{r}\left(t^{\prime}\right)=(1 / r) z^{r}\left(r^{\beta} t^{\prime}\right)$. For each $t^{\prime}>0, \bar{z}^{r}\left(t^{\prime}\right)$ is an infinitely divisible random variable, whose Lévy measure $\bar{\mu}^{r}(d \zeta)=$ $1_{\{|\zeta| \leq 1\}} \mu(r d \zeta)$ at $t^{\prime}=1$ satisfies assumptions (A.1) and (A.2). We introduce

$$
\Theta_{t}=\frac{\partial \gamma}{\partial \zeta}(x, 0) \bar{z}^{r}(1)=\frac{\partial \gamma}{\partial \zeta}(x, 0)\left(t^{-1 / \beta} z^{r}(t)\right) .
$$

Then $\mathcal{X}_{t}-\Theta_{t}$ converges in $L^{1}$ to 0 as $t \rightarrow 0$ (to $b(x)$ in case $\beta=1$ ) (cf. [20, Lemma 7]). Furthermore $|(\partial \gamma / \partial \zeta)(x, 0)|$ is bounded and $((\partial \gamma / \partial \zeta)(x, 0))((\partial \gamma / \partial \zeta)(x, 0))^{*}$ is uniformly elliptic by the assumption (A.3). Hence by Lemma 3.3, we conclude the density of $\mathcal{X}_{t}$ is bounded away from 0 at zero as $t \rightarrow 0$.

4. Proof of Theorem 2. (a) For $n \geq \alpha(x, y)$ we have $P\left(\left|U_{n}-y\right| \leq \varepsilon\right) \geq c$ for $\varepsilon \in(0,1]$, since $\mu$ has point masses. Hence $p_{t}(x, y) \geq C t^{\alpha(x, y)-d / \beta}$ by Lemma 2.1.

For the upper bound, if $n<\alpha(x, y)$, then by choosing $K_{n}$ small, we have $y \notin Q_{x}^{(n)}$. That is,

$$
P\left(\left|V_{n}-y\right| \leq \varepsilon\right)=0 \text { for } \varepsilon>0 \text { small, }
$$

and we may choose $\gamma_{n}=+\infty$ in (2.4) and (2.5). On the other hand, if $n \geq \alpha(x, y)$, then we must choose $\gamma_{n}=0$ in (2.4) $\left(\varphi_{n}=i d\right.$ must satisfy it).

Hence we may choose $\Gamma=\alpha(x, y)-d / \beta$ in the conclusion. These imply

$$
p_{t}(x, y) \asymp t^{\alpha(x, y)-d / \beta} .
$$

(b) We set $\mathcal{S}_{t, k}=\left([0, t]^{k} / \sim\right)$ and $\mathcal{S}_{t}=\bigsqcup_{k \geq 0} \mathcal{S}_{t, k}$, where $\bigsqcup_{k \geq 0}$ denotes the disjoint sum and $\sim$ means the identification of the coordinates on the product space $[0, t]^{k}$ by the permutation. 
Let $r=r(t)=t^{1 / \beta}$. The distribution $\tilde{P}_{t, r}$ of the moments (instants) of jumps related to $\tilde{z}^{r}(s)$ during $[0, t]$ is given by

$$
\begin{aligned}
\int_{\left\{\# S_{t}=k\right\}} & f\left(S_{t}\right) d \tilde{P}_{t, r}\left(S_{t}\right) \\
= & \left\{\left(t \int 1_{\{|\zeta|>r\}}(\zeta) \mu(d \zeta)\right)^{k}\left(\frac{1}{k !}\right) \exp \left(-t \int 1_{\{|\zeta|>r\}}(\zeta) \mu(d \zeta)\right)\right\} \\
& \times \frac{1}{t^{k}} \int_{0}^{t} \cdots \int_{0}^{t} f\left(s_{1}, \ldots, s_{k}\right) d s_{1} \cdots d s_{k},
\end{aligned}
$$

where $f$ is a function on $\mathcal{S}_{t, k}$ (a symmetric function on $[0, t]^{k}$ ). Given $S_{t} \in \mathcal{S}_{t}$, we introduce the process $x_{s}\left(r, S_{t}, x\right)$ as the solution of the following S.D.E.:

$$
\begin{aligned}
x_{s}\left(r, S_{t}, x\right)= & x-\int_{0}^{s} d u \int 1_{\{|\zeta|>r\}}(\zeta) \gamma\left(x_{u}\left(r, S_{t}, x\right), \zeta\right) \mu^{r}(d \zeta) \\
& +\sum_{u \leq s}^{c} \gamma\left(x_{u^{-}}\left(r, S_{t}, x\right), \Delta z^{r}(u)\right)+\sum_{s_{i} \in S_{t}, s_{i} \leq s} \gamma\left(x_{s_{i}-}\left(r, S_{t}, x\right), \xi_{i}^{r}\right),
\end{aligned}
$$

where $\left(\xi_{n}^{r}\right)_{n \in N}$ denotes a series of random variables (i.i.d.) obeying the probability law

$$
\mu^{r}(d \zeta)=\frac{1_{\{|\zeta|>r\}}(\zeta) \cdot \mu(d \zeta)}{\left.\int 1_{\{|\zeta|>r\}}(\zeta) \cdot \mu(d \zeta)\right)}
$$

We remark that $x_{s}\left(r, S_{t}, x\right)$ is a martingale for each $0<r<1$, due to the assumption $b(x) \equiv$ 0 . We define a new Markov chain $\left(U_{n}^{r}\right)_{n \in N}$ by $U_{0}^{r}=x$ and $U_{n+1}^{r}=U_{n}^{r}+\gamma\left(U_{n}^{r}, \xi_{n+1}^{r}\right), n \in N$.

We can prove due to Proposition 1.1 that under (A.1) through (A.4) the law of $x_{s}\left(r, S_{t}, x\right)$ for $\left(d s\right.$-a.e.) $s>0$ has a $C_{b}^{\infty}$-density, denoted by $p_{s}\left(r, S_{t}, x, y\right)$.

Indeed, let $0 \leq s \leq t$. In the case $S_{t}=\emptyset$, we can choose $1_{\{|\zeta| \leq r\}} \cdot \mu(d \zeta)$ for the measure $\mu(d \zeta)$ in Proposition 1.1. Hence we have the existence of the density for the law $p_{s}(r, \emptyset, x, d y)$ of $x_{s}(r, \emptyset, x)\left(=\left.x_{s}\left(r, S_{t}, x\right)\right|_{S_{t}=\emptyset}\right): p_{s}(r, \emptyset, x, d y)=p_{s}(r, \emptyset, x, y) d y$.

Next we consider the general case. Since $\tilde{z}^{r}(s)$ and $z^{r}(s)$ are independent, we have by Markov property that the law $p_{s}\left(r, S_{t}, x, d y\right) \equiv P\left(x_{s}\left(r, S_{t}, x\right) \in d y\right)$ of $x_{s}\left(r, S_{t}, x\right)$ is represented by

$$
\begin{aligned}
p_{s}\left(r, S_{t}, x, d y\right) \\
=\int d z_{0}^{\prime} \int_{P_{z_{0}^{\prime}}} p_{s_{1}}\left(r, \emptyset, x, z_{0}^{\prime}\right) g_{r}\left(z_{0}^{\prime}, d z_{1}\right) \int d z_{1}^{\prime} \int_{P_{z_{1}^{\prime}}} p_{s_{2}-s_{1}}\left(r, \emptyset, z_{1}, z_{1}^{\prime}\right) g_{r}\left(z_{1}^{\prime}, d z_{2}\right) \\
\quad \ldots \int d z_{n_{1}-1}^{\prime} \int_{P_{z_{n_{1}}^{\prime}-1}}\left\{p_{s_{n_{1}}-s_{n_{1}-1}}\left(r, \emptyset, z_{n_{1}-1}, z_{n_{1}-1}^{\prime}\right) g_{r}\left(z_{n_{1}-1}^{\prime}, d z_{n_{1}}\right) p_{t-s_{n_{1}}}\left(r, \emptyset, z_{n_{1}}, d y\right)\right\}
\end{aligned}
$$

if $S_{t} \in \mathcal{S}_{t, n_{1}}$ (cf. [8, (2.7)]). Here $g_{r}(x, d z)=P\left(x+\gamma\left(x, \xi_{i}^{r}\right) \in d z\right)$.

On the other hand, we have by the independence of $\tilde{z}^{r}(s)$ and $z^{r}(s)$ again

$$
p_{s}(x, d y)=\int_{\mathcal{S}_{t}} p_{s}\left(r, S_{t}, x, d y\right) d \tilde{P}_{t, r}\left(S_{t}\right),
$$


using the factorization of the measure $N$ (cf. [11, p. 71]). By Proposition 1.1, L.H.S. has the density $p_{s}(x, y)$ with respect to $d y$. Hence $p_{s}\left(r, S_{t}, x, d y\right)$ is absolutely continuous with respect to $d y\left(d \tilde{P}_{t, r}\right.$-a.s.). Hence we have by the derivation under the integral sign

$$
p_{s}(x, y)=\int_{\mathcal{S}_{t}}\left(p_{s}\left(r, S_{t}, x, d y\right) / d y\right)(y) d \tilde{P}_{t, r}\left(S_{t}\right) .
$$

We denote by $p_{s}\left(r, S_{t}, x, y\right)$ the derivative $p_{s}\left(r, S_{t}, x, d y\right) / d y(y)$ which is defined uniquely $d \tilde{P}_{t, r} \otimes d y$-a.e. (Since $\left.d \tilde{P}_{t, r}\right|_{\mathcal{S}_{t, k}}$ is the uniform distribution on $\mathcal{S}_{t, k}, p_{s}\left(r, S_{t}, x, y\right)$ is defined uniquely $d s \otimes d y$-a.e.) Since $y \mapsto p_{s}(x, y)$ is smooth, so is $y \mapsto p_{s}\left(r, S_{t}, x, y\right) d s$-a.s., and hence $p_{s}\left(r, S_{t}, x, y\right)$ is defined as a smooth density $d s$-a.e.

Thus by taking $s=t$

$$
p_{t}(x, y)=\int_{\mathcal{S}_{t}} p_{t}\left(r, S_{t}, x, y\right) \tilde{P}_{t, r}\left(S_{t}\right)=\sum_{k=0}^{\infty} p_{t}(k, r, x, y),
$$

where

$$
p_{t}(k, r, x, y)=\int_{\mathcal{S}_{t, k}} p_{t}\left(r, S_{t}, x, y\right) d \tilde{P}_{t, r}\left(S_{t}\right)
$$

Hence

$$
\begin{aligned}
p_{t}(x, y) d y & =E^{\tilde{P}_{t, r}\left[P\left(x_{t}\left(r, S_{t}, x\right) \in d y\right)\right]} \\
& =E^{\tilde{P}_{t, r}} E^{\left(\mu^{r}\right)^{\otimes \# S_{t}}}\left[P\left(x_{t}\left(r, S_{t}, x\right) \in d y \mid S_{t}, \xi_{1}^{r}, \ldots, \xi_{\# S_{t}}^{r}\right)\right] .
\end{aligned}
$$

For each $S_{t} \in \mathcal{S}_{t}$, we put $n_{1}=k$ if $S_{t} \in \mathcal{S}_{t, k}$. We then have

$$
\begin{aligned}
E^{\left(\mu^{r}\right)^{\otimes \# S_{t}}}\left[P\left(x_{t}\left(r, S_{t}, x\right) \in d y\right) \mid S_{t}\right] \\
=E^{\left(\mu^{r}\right)^{\otimes \# S_{t}}}\left[P\left(x_{t}\left(r, S_{t}, x\right) \in d y:\left|y-U_{n_{1}}^{r}\right| \leq t^{1 / \beta^{\prime}} \mid S_{t}, \xi_{1}^{r}, \ldots, \xi_{n_{1}}^{r}\right)\right] \\
\quad+E^{\left(\mu^{r}\right)^{\otimes \# S_{t}}}\left[P\left(x_{t}\left(r, S_{t}, x\right) \in d y:\left|y-U_{n_{1}}^{r}\right|>t^{1 / \beta^{\prime}} \mid S_{t}, \xi_{1}^{r}, \ldots, \xi_{n_{1}}^{r}\right)\right] .
\end{aligned}
$$

First we have to compute $\boldsymbol{P}\left(\left|y-U_{n_{1}}^{r}\right| \leq t^{1 / \beta^{\prime}}\right) \equiv E^{\left(\mu^{r}\right)^{\otimes n_{1}}}\left[P\left(\left|y-U_{n_{1}}^{r}\right| \leq t^{1 / \beta^{\prime}} \mid \xi_{1}^{r}, \ldots\right.\right.$, $\left.\xi_{n_{1}}^{r}\right)$ ] for a given $y=x_{t}\left(r, S_{t}, x\right)$ with $S_{t} \in \mathcal{S}_{t, n_{1}}$. We denote by the random variable $W_{n}$ the number of $a_{n}$ in $\left(\xi_{i}^{r}\right)_{i=1}^{n_{1}}$.

Given $n_{1} \in N$, let $\left(w_{n}\right)_{n=0}^{N}$ and $w_{n} \in N \cup\{0\}$ be a series of integers such that $n_{1}=$ $\sum_{n=0}^{N} w_{n}$. We then have

$$
\begin{aligned}
& \boldsymbol{P}\left(\text { for all } n \leq N, \quad W_{n}=w_{n} \text { and }\left|y-U_{n_{1}}^{r}\right| \leq t^{1 / \beta^{\prime}}\right) \\
& \quad \leq \prod_{n=0}^{N} \frac{1}{w_{n} !}\left(t k_{n}\right)^{w_{n}} e^{-t k_{n}},
\end{aligned}
$$


since each $W_{n}$ is a Poisson random variable with mean $t k_{n}$. Hence

$$
\begin{gathered}
\left.\log \boldsymbol{P} \text { (for all } n \leq N, W_{n}=w_{n} \text { and }\left|y-U_{n_{1}}^{r}\right| \leq t^{1 / \beta^{\prime}}\right) \\
\leq-\sum_{n=0}^{N}\left(w_{n} \log \left(1 /\left(t k_{n}\right)\right)+\log \left(w_{n} !\right)+t k_{n}\right) \\
\quad=-\sum_{n=0}^{N}\left(w_{n} \log \left(1 /\left(t k_{n}\right)\right)+\log \left(w_{n} !\right)\right)+O(1)
\end{gathered}
$$

We introduce the set $\mathcal{W}$ of all $\left(w_{n}\right)_{n=0}^{N}$ 's such that for some $n_{1} \in N, U_{n_{1}}^{r}$ directed by the following condition (*) satisfies $\left|y-U_{n_{1}}^{r}\right| \leq t^{1 / \beta^{\prime}}$ :

(*) $\quad w_{n}=\left(\#\right.$ of $a_{n}$ 's which appear in $\left.\xi_{1}^{r}, \ldots, \xi_{n_{1}}^{r}\right)$ and $n_{1}=\sum_{n=1}^{N} w_{n}$.

Then it follow from (4.7) that

$$
\begin{array}{r}
\log \boldsymbol{P}\left(\begin{array}{c}
\text { there exists }\left(w_{n}\right) \in \mathcal{W} \text { such that for all } n \leq N, \\
W_{n}=w_{n} \text { and }\left|y-U_{n_{1}}^{r}\right| \leq t^{1 / \beta^{\prime}}
\end{array}\right) \\
\leq-\min _{\mathcal{W}} \sum_{n=0}^{N}\left(w_{n} \log \left(1 /\left(t k_{n}\right)\right)+\log \left(w_{n} !\right)\right)+O(\log |\mathcal{W}|) .
\end{array}
$$

On the other hand, we have

$$
\boldsymbol{P}\left(W_{n} \geq N^{3} \text { and }\left|y-U_{n_{1}}^{r}\right| \leq t^{1 / \beta^{\prime}}\right) \leq C e^{-N^{3}} \leq C e^{-c(\log (1 / t))^{3}},
$$

since $W_{n}$ is a Poisson variable with mean $t k_{n}$. Since this is very small relative to the probability above, we may put the restriction $w_{n} \leq N^{3}$ and $n_{1} \leq N^{4}$. Hence we may write $|\mathcal{W}|=O\left(\left(N^{3}\right)^{N}\right)$, and

$$
\log |\mathcal{W}|=O(N \log N)=O(\log (1 / t) \log \log (1 / t)) .
$$

Let $x_{t}(r, \emptyset, x)$ denote the process defined by (4.3) with $S_{t}=\emptyset$, and $p_{t}(r, \emptyset, x, y)$ its density. By Theorem 1(a), $p_{t}(r, \emptyset, x, y) \leq C t^{-d / \beta}$ as $t \rightarrow 0$, and this implies, for given $S_{t} \in \mathcal{S}_{t, n_{1}}, \xi_{1}^{r}, \ldots, \xi_{n_{1}}^{r}$, that $P\left(x_{t}\left(r, S_{t}, x\right) \in d y \mid S_{t}, \xi_{1}^{r}, \ldots, \xi_{n_{1}}^{r}\right) / d y=O\left(t^{-d / \beta}\right)$ as $t \rightarrow 0$. Since $z^{r}(u)$ and $\left(\xi_{i}^{r}\right)$ are independent, by (4.4) and Fubini's Theorem we have

$$
\begin{aligned}
\log \left(E^{\tilde{P}_{t, r} \mid \mathcal{S}_{t, n_{1}}} E^{\left(\mu^{r}\right)^{\otimes n_{1}}}\left[P\left(x_{t}\left(r, S_{t}, x\right) \in d y:\left|y-U_{n_{1}}^{r}\right| \leq t^{1 / \beta^{\prime}} \mid S_{t}, \xi_{1}^{r}, \ldots, \xi_{n_{1}}^{r}\right)\right] / d y\right) \\
\leq \log \left(t ^ { - d / \beta } \operatorname { e x p } \left(-\min _{\mathcal{W}} \sum_{n=0}^{N}\left(w_{n} \log \left(1 /\left(t k_{n}\right)\right)\right.\right.\right. \\
\left.\left.\left.\quad+\log \left(w_{n} !\right)\right)+O(\log (1 / t) \log \log (1 / t))\right)\right) \\
\leq-\min _{\mathcal{W}} \sum_{n=0}^{N}\left(w_{n} \log \left(1 /\left(t k_{n}\right)\right)+\log \left(w_{n} !\right)\right)+O(\log (1 / t) \log \log (1 / t)) .
\end{aligned}
$$

For the second term, we have the following lemma, whose proof is given in Appendix. 
LEMMA 4.1. Given $y=x_{t}\left(r, S_{t}, x\right), S_{t} \in \mathcal{S}_{t, n_{1}}$ and $U_{n_{1}}^{r}=A_{n_{1}}\left(x, \xi_{1}^{r}, \ldots, \xi_{n_{1}}^{r}\right)$, there exist $k>0$ and $C_{0}>0$ such that for every $p>k$

$$
\begin{aligned}
& E^{\tilde{P}_{t, r} \mid \mathcal{S}_{t, n_{1}}} E^{\left(\mu^{r}\right)^{\otimes n_{1}}}\left[P\left(\left|y-U_{n_{1}}^{r}\right|>t^{1 / \beta^{\prime}} \mid S_{t}, \xi_{1}^{r}, \ldots, \xi_{n_{1}}^{r}\right)\right] \\
& \leq n_{1} C_{0} \exp \left[-(p-k)(\log (1 / t))^{2}\right]
\end{aligned}
$$

as $t \rightarrow 0$.

Given $\xi_{1}^{r}, \ldots, \xi_{n_{1}}^{r}$, we have as above $E^{\tilde{P}_{t, r} \mid \mathcal{S}_{t, n_{1}}}\left[P\left(x_{t}\left(r, S_{t}, x\right) \in d y \mid S_{t}, \xi_{1}^{r}, \ldots, \xi_{n_{1}}^{r}\right) / d y\right]$ $=O\left(t^{-d / \beta}\right)$ as $t \rightarrow 0$. We integrate this with respect to $\left(\mu^{r}\right)^{\otimes n_{1}}$ on $\left\{\left|y-U_{n_{1}}^{r}\right|>t^{1 / \beta^{\prime}}\right\}$. Since $z^{r}(u)$ and $\left(\xi_{i}^{r}\right)$ are independent, we then have by Lemma 4.1

$$
\begin{aligned}
& E^{\tilde{P}_{t, r} \mid \mathcal{S}_{t, n_{1}}} E^{\left(\mu^{r}\right)^{\otimes n_{1}}}\left[P\left(x_{t}\left(r, S_{t}, x\right) \in d y:\left|y-U_{n_{1}}^{r}\right|>t^{1 / \beta^{\prime}} \mid S_{t}, \xi_{1}^{r}, \ldots, \xi_{n_{1}}^{r}\right)\right] / d y \\
& \quad \leq n_{1} C_{0}^{\prime} t^{-d / \beta} \exp \left[-(p-k)(\log (1 / t))^{2}\right],
\end{aligned}
$$

as $t \rightarrow 0$.

We get

$$
\begin{aligned}
& \log \left(E^{\left.\tilde{P}_{t, r}\right|_{\mathcal{S}_{t, n_{1}}}} E^{\left(\mu^{r}\right)^{\otimes n_{1}}}\left[P\left(x_{t}\left(r, S_{t}, x\right) \in d y:\left|y-U_{n_{1}}^{r}\right|>t^{1 / \beta^{\prime}} \mid S_{t}, \xi_{1}^{r}, \ldots, \xi_{n_{1}}^{r}\right)\right] / d y\right) \\
& \quad \leq-(p-k)(\log (1 / t))^{2}+\max _{n_{1} \leq N^{4}} \log n_{1}+\log C_{0}^{\prime}+O(\log (1 / t)) \\
& \quad \leq-(p-k)(\log (1 / t))^{2}+O(\log (1 / t))
\end{aligned}
$$

since $\max _{n_{1} \leq N^{4}} \log n_{1}=\log N^{4}=O(\log (1 / t))$. Since $p>k$ is arbitrary, this can be neglected in view of R.H.S. of (4.10) and (4.6).

After summing up $E^{\tilde{P}_{t, r} \mid \mathcal{S}_{t, n_{1}}} E^{\left(\mu^{r}\right)^{\otimes n_{1}}}[\ldots]$ with respect to $n_{1}=0, \ldots, N^{4}$, we get

$$
\begin{aligned}
\log \left(E^{\tilde{P}_{t, r} \mid \cup_{k \leq N^{4}} \mathcal{S}_{t, k}} E^{\left(\mu^{r}\right)^{\otimes \# S_{t}}}\left[P\left(x_{t}\left(r, S_{t}, x\right) \in d y \mid S_{t}, \xi_{1}^{r}, \ldots, \xi_{\# S_{t}}^{r}\right)\right] / d y\right) \\
\leq-\min _{\mathcal{W}} \sum_{n=0}^{N}\left(w_{n} \log \left(1 /\left(t k_{n}\right)\right)+\log \left(w_{n} !\right)\right) \\
\quad+O(\log (1 / t) \log \log (1 / t))+O(\log (1 / t)) .
\end{aligned}
$$

In view of (4.5) and (4.9), we get

$$
\log p_{t}(x, y) \leq-\min _{\mathcal{W}} \sum_{n=0}^{N}\left(w_{n} \log \left(1 /\left(t k_{n}\right)\right)+\log \left(w_{n} !\right)\right)+O(\log (1 / t) \log \log (1 / t)) .
$$

Since there is no difference between the trajectories of the deterministic chain $A_{n_{1}}\left(x, \xi_{1}, \ldots\right.$, $\left.\xi_{n_{1}}\right)$ and $U_{n_{1}}^{r}$ obtained by using $\left\{a_{n} ; n=0, \ldots, N\right\}$ under (1.14), we have the assertion.

5. Appendix. Proof of Lemma 4.1. We fix $0<r<1$, and put $\xi(r, \cdot, x) \equiv x+$ $\gamma(x, \cdot)$ when $\cdot$ is occupied by the random variables $\xi_{i}^{r}$.

Choose $S_{t}=\emptyset$ and consider the process $s \mapsto x_{s}(r, \emptyset, x)$ as the solution of (4.3). Given $\left(s_{1}, y\right)$, the solution for $s_{2} \geq s_{1}$ with the initial value $z$ at $s=s_{1}$ is given by a smooth 
stochastic semiflow $\phi_{s_{1}, s_{2}}(z)$. This is proved in [4]. Assuming $y=x_{t}\left(r, S_{t}, x\right), S_{t} \in \mathcal{S}_{t, n_{1}}$, we shall estimate $\left|U_{n_{1}}^{r}-x_{t}\left(r, S_{t}, x\right)\right|$, and show that for $p>k$ and $t \leq 1$

$$
\begin{aligned}
& E^{\tilde{P}_{t, r} \mid \mathcal{S}_{t, n_{1}}} E^{\left(\mu^{r}\right)^{\otimes n_{1}}}\left[P\left(\left|U_{n_{1}}^{r}-x_{t}\left(r, S_{t}, x\right)\right|>t^{1 / \beta^{\prime}} \mid S_{t}, \xi_{1}^{r}, \ldots, \xi_{n_{1}}^{r}\right)\right] \\
& \quad \leq n_{1} C_{0} \exp \left[-(p-k)(\log (1 / t))^{2}\right] .
\end{aligned}
$$

We first recall that $U_{n_{1}}^{r}$ and $x_{t}\left(r, S_{t}, x\right)$ can be represented by

$$
U_{n_{1}}^{r}=A_{n_{1}}\left(x, \xi_{1}^{r}, \ldots, \xi_{n_{1}}^{r}\right)=\xi\left(r, \xi_{n_{1}}^{r}, \cdot\right) \circ \xi\left(r, \xi_{n_{1}-1}^{r}, \cdot\right) \circ \cdots \circ \xi\left(r, \xi_{n_{1}}^{r}, x\right)
$$

and

$$
x_{t}\left(r, S_{t}, x\right)=\phi_{t_{n_{1}}, t} \circ \xi\left(r, \xi_{n_{1}}^{r}, \cdot\right) \circ \phi_{t_{n_{1}-1}, t_{n_{1}}} \circ \cdots \circ \xi\left(r, \xi_{2}^{r}, \cdot\right) \circ \phi_{t_{1}, t_{2}} \circ \xi\left(r, \xi_{1}^{r}, \cdot\right) \circ \phi_{0, t_{1}}(x) .
$$

(1) Case $n_{1}=0$. In this case, $S_{t}=\emptyset$ and $U_{n_{1}}^{r}=x$. The process $x_{t}(r, \emptyset, x)$ is a martingale, whose quadratic variation $[x(r, \emptyset, x), x(r, \emptyset, x)]_{t}$ is bounded by

$$
\int_{0}^{t} d u \int_{-r}^{r}\left\{\gamma\left(x_{u-}(r, \emptyset, x), \zeta\right)\right\}^{2} \mu(d \zeta) \leq t \cdot\left(2 \sup _{x, \zeta}\left|\frac{\partial \gamma}{\partial \zeta}(x, \zeta)\right|\right)^{2} \cdot \int\left(\zeta^{2} \wedge 1\right) \mu(d \zeta) \leq k t
$$

for some $k>0$, which is uniform in $0<r<1$ and $x$. We have that, for given $p>k$ and $t \leq 1$,

$$
P\left(\sup _{0 \leq s \leq t, x}\left|x_{s}(r, \emptyset, x)-x\right|>t^{1 / \beta^{\prime}}\right) \leq C_{0} \exp \left[-(p-k)(\log (1 / t))^{2}\right]
$$

with $C_{0}$ not depending on $x, r$, and hence the assertion follows for this case.

Indeed, by the upper bound of exponential type [16, Theoreme 13],

$$
P\left(\sup _{0 \leq s \leq t, x}\left|x_{s}(r, \emptyset, x)-x\right| \geq C\right) \leq 2 \exp \left[-\lambda C+\frac{1}{2}(\lambda S)^{2} k t(1+\exp \lambda S)\right]
$$

for $C>0, \lambda>0$. We choose $C=p S \log (1 / t)$ and $\lambda=(\log (1 / t)) / S$. Then

$$
\begin{aligned}
& P\left(\sup _{0 \leq s \leq t, x}\left|x_{s}(r, \emptyset, x)-x\right| \geq p S \log (1 / t)\right) \\
& \quad \leq 2 \exp \left[-p(\log (1 / t))^{2}+(\log (1 / t))^{2} k t+k(\log (1 / t))^{2}\right] \\
& \quad \leq C_{0} \exp \left[-(p-k)(\log (1 / t))^{2}\right] \text { as } t \rightarrow 0 .
\end{aligned}
$$

We choose $S=(1 / p) t^{1 / \beta}$. Then

$$
\begin{aligned}
& P\left(\sup _{0 \leq s \leq t, x}\left|x_{s}(r, \emptyset, x)-x\right| \geq t^{1 / \beta^{\prime}}\right) \\
& \quad \leq P\left(\sup _{0 \leq s \leq t, x}\left|x_{s}(r, \emptyset, x)-x\right| \geq t^{1 / \beta} \log (1 / t)\right) \\
& \quad \leq C_{0} \exp \left[-(p-k)(\log (1 / t))^{2}\right],
\end{aligned}
$$

as $t \rightarrow 0$. Here $\beta^{\prime}>\beta$ and the constant $C_{0}$ does not depend on $x, r$. 
(2) Case $n_{1} \geq 1$. Assume $S_{t}=\left\{t_{1}, \ldots, t_{n_{1}}\right\}$ with $t_{1}<\cdots<t_{n_{1}}$. Given $\xi_{1}^{r}, \ldots, \xi_{n_{1}}^{r}$, we put

$$
I_{n_{1}} \equiv U_{n_{1}}^{r}-x_{t}\left(r, S_{t}, x\right)
$$

and

$$
M \equiv \max \left(\sup _{x, \zeta}\left\|\frac{\partial \xi}{\partial x}(r, \zeta, x)\right\|, 1\right)=\max \left(\sup _{x, \zeta}\left\|I+\frac{\partial \gamma}{\partial x}(x, \zeta)\right\|, 1\right),
$$

which exists by the assumption on $\gamma$. Here $\|\cdot\|$ denotes the norm of the matrix. In rewriting (a.5), we have by an elementaly calculation

$$
\begin{aligned}
\left|I_{n_{1}}\right| \leq & \sup _{y}\left|\phi_{t_{n_{1}}, t}(y)-y\right|+M \sup _{y}\left|\phi_{t_{n_{1}-1}, t_{n_{1}}}(y)-y\right| \\
& +M^{2} \sup _{y}\left|\phi_{t_{n_{1}-2}, t_{n_{1}-1}}(y)-y\right|+\cdots+M^{n_{1}} \sup _{x}\left|\phi_{0, t_{1}}(x)-x\right| .
\end{aligned}
$$

We assume $\left|I_{n_{1}}\right|>t^{1 / \beta^{\prime}}$. Then for some $j \in\left\{1, \ldots, n_{1}\right\}$,

$$
\sup _{y}\left|\phi_{t_{j-1}, t_{j}}(y)-y\right|>\left(1 / n_{1}\right) M^{-n_{1}} t^{1 / \beta^{\prime}} .
$$

Indeed, if for all $j, \sup _{y}\left|\phi_{t_{j-1}, t_{j}}(y)-y\right| \leq\left(1 / n_{1}\right) M^{-n_{1}} t^{1 / \beta^{\prime}}$, then

$$
\begin{aligned}
\left|I_{n_{1}}\right| \leq & \sup _{y}\left|\phi_{t_{n_{1}}, t}(y)-y\right|+M \sup _{y}\left|\phi_{t_{n_{1}-1}, t_{n_{1}}}(y)-y\right| \\
& +M^{2} \sup _{y}\left|\phi_{t_{n_{1}-2}, t_{n_{1}-1}}(y)-y\right|+\cdots+M^{n_{1}} \sup _{x}\left|\phi_{0, t_{1}}(x)-x\right| \\
\leq & \left(1 / n_{1}\right) M^{-n_{1}} t^{1 / \beta^{\prime}}+\left(1 / n_{1}\right) M^{-n_{1}+1} t^{1 / \beta^{\prime}}+\cdots+\left(1 / n_{1}\right) t^{1 / \beta^{\prime}} \leq t^{1 / \beta^{\prime}},
\end{aligned}
$$

which is a contradiction. Hence

$$
\begin{aligned}
& E^{\tilde{P}_{t, r} \mid \mathcal{S}_{t, n_{1}}} E^{\left(\mu^{r}\right)^{\otimes n_{1}}}\left[P\left(\left|I_{n_{1}}\right|>t^{1 / \beta^{\prime}}\left|S_{t}, \xi_{1}^{r}, \ldots, \xi_{n_{1}}^{r}\right| S_{t}\right)\right] \\
& \quad \leq E^{\tilde{P}_{t, r} \mid \mathcal{S}_{t, n_{1}}}\left[P\left(\text { there exists } j \text { such that } \sup _{y}\left|\phi_{t j-1}, t_{j}(y)-y\right|>\left(1 / n_{1}\right) M^{-n_{1}} t^{1 / \beta^{\prime}}\right)\right] \\
& \quad \leq n_{1} P\left(\sup _{0 \leq s \leq t, x}\left|x_{s}(r, \emptyset, x)-x\right|>\left(1 / n_{1}\right) M^{-n_{1}} t^{1 / \beta^{\prime}}\right) .
\end{aligned}
$$

We choose $\beta^{\prime \prime}=\beta^{\prime \prime}\left(n_{1}\right)$ such that $\beta<\beta^{\prime \prime}<\beta^{\prime}$ and $t^{1 / \beta}<t^{1 / \beta^{\prime \prime}}<\left(1 / n_{1}\right) M^{-n_{1}} t^{1 / \beta^{\prime}}<$ $t^{1 / \beta^{\prime}}$ for $t>0$ small. Then by the proof of (a.2)

$$
\begin{aligned}
& E^{\tilde{P}_{t, r} \mid \mathcal{S}_{t, n_{1}}} E^{\left(\mu^{r}\right)^{\otimes n_{1}}}\left[P\left(\left|I_{n_{1}}\right|>t^{1 / \beta^{\prime}} \mid S_{t}, \xi_{1}^{r}, \ldots, \xi_{n_{1}}^{r}\right)\right] \\
& \quad \leq n_{1} P\left(\sup _{0 \leq s \leq t, x}\left|x_{s}(r, \emptyset, x)-x\right|>t^{1 / \beta^{\prime \prime}}\right) \\
& \quad \leq n_{1} C_{0} \exp \left[-(p-k)(\log (1 / t))^{2}\right]
\end{aligned}
$$

as $t \rightarrow 0$. This proves (a.1). 


\section{REFERENCES}

[ 1 ] K. Bichteler, J.-B. Gravereaux And J. Jacod, Malliavin calculus for processes with jumps, Stochastics Monogr. 2, Gordon and Breach Science Publishers, New York, 1987.

[ 2 ] K. BiChTEler AND J. JACOD, Calcul de Malliavin pour les diffusions avec sauts: existence d'une densité dans le case unidimensionel, in Séminaire de Probabilités XVII (J. Azéma and M. Yor, eds.), 132-157, Lecture Notes in Math. 986, Springer-Verlag, Berlin, 1983.

[ 3 ] J.-M. BISmUT, Calcul des variations stochastiques et processus de sauts, Z. Wahrsch. Verw. Gebiete 63 (1983), 147-235.

[ 4 ] T. FUJIWARA AND H. KUNITA, Stochastic differential equations for jump type and Lévy processes in diffeomorphisms group, J. Math. Kyoto Univ. 25 (1985), 71-106.

[ 5 ] W. HOH AND N. JACOB, Some Dirichlet forms generated by pseudo differential operators, Bull. Sci. Math. 116 (1992), 383-398.

[ 6 ] N. IKEDA AND S. Watanabe, Stochastic differential equations and diffusion processes, North-Holland, Amsterdam-New York; Kodansha, Tokyo, 1981.

[ 7 ] Y. IshiKawA, On the lower bound of the density for jump processes in small time, Bull. Sci. Math. 117 (1993), 463-483.

[ 8 ] Y. IsHIKAWA, Asymptotic behavior of the transition density for jump-type processes in small time, Tohoku Math. J. 46 (1994), 443-456.

[ 9 ] Y. IshiKaWA, Large deviation estimate of transition densities for jump processes, Ann. Inst. H. Poincaré Probab. Statist. 33 (1997), 179-222.

[10] Y. IsHIKAWA, On the upper bound of the density for truncated stable processes in small time, Potential Anal. 6 (1997), 11-37.

[11] J. JACOD, Calcul stochastique et problèm des martingales, Lecture Notes in Math. 714, Springer-Verlag, Berlin, 1975.

[12] H. KUNITA, Stochastic differential equations with jumps and stochastic flows of diffeomorphisms, in Itô's stochastic calculus and probability theory (N. Ikeda, S. Watanabe, M. Fukushima and H. Kunita, eds.), 197-211, Springer-Verlag, Tokyo-Berlin, 1996.

[13] R. LÉANDRE, Densité en temps petit d'un processus de sauts, in Séminaire de Probabilités XXI (J. Azéma, P. A. Meyer and M. Yor, eds.), 81-99, Lecture Notes in Math. 1247, Springer-Verlag, Berlin, 1987.

[14] R. LÉAndre, Régularité de processus de sauts dégénérés (II), Ann. Inst. H. Poincaré Probab. Statist. 24 (1988), 209-236.

[15] Z-M. MA, M. Röckner AND T-S. Zhang, Approximation of arbitrary Dirichlet processes by Markov chains, Ann. Inst. H. Poincaré Probab. Statist. 34 (1998), 1-22.

[16] J. P. LEPELTIER AND R. MARCHAL, Problèmes de martingales associées à un opérateur intégro-différentiel, Ann. Inst. H. Poincaré Probab. Statist. 12 (1976), 43-103.

[17] J. R. NoRRIS, Integration by parts for jump processes, in Séminaire de Probabilités XXII (J. Azéma, P. A Meyer and M. Yor, eds.), 271-315, Lecture Notes in Math. 1321, Springer-Verlag, Berlin, 1988.

[18] J. PICARD, On the existence of smooth densities for jump processes, Probab. Theory Related Fields 105 (1996), 481-511.

[19] J. PICARD, Density in small time for Lévy processes, ESAIM Probab. Statist. 1 (1997), 358-389 (electronic).

[20] J. PICARD, Density in small time at accessible points for jump processes, Stochastic Process. Appl. 67 (1997), 251-279.

[21] N. PRIVAULT, Linear Skorohod stochastic differential equations on Poisson space, in Stochastic analysis and related topics V (H. Körezlioglu, B. Oksendal and A. S. Üstünel, eds.), 237-253, Progr. Probab. 38, Birkhäuser, Boston-Basel-Berlin, 1996.

[22] P. ProtTER, Stochastic Integration and Differential Equations, Springer-Verlag, Berlin, 1990.

[23] M. TSUCHIYA, Lévy measure with generalized polar decomposition and the associated SDE with jumps, Stochastics Stochastic Rep. 38 (1992), 95-117. 
DEPARTMENT OF MATHEMATICS

FACULTY OF SCIENCE

EHIME UNIVERSITY

MATSUYAMA, EhIME 7908577

JAPAN

e-mail address: slishi@math.sci.ehime-u.ac.jp 